\title{
Rhabdomyolysis-induced acute kidney injury in a cancer patient exposed to denosumab and abiraterone: a case report CrossMark
}

Javier A. Neyra ${ }^{1,2^{*}}$, Natalia A. Rocha ${ }^{1}$, Rhea Bhargava ${ }^{4}$, Omkar U. Vaidya ${ }^{4,5}$, Allen R. Hendricks ${ }^{3}$ and Aylin R. Rodan ${ }^{1,2}$

\begin{abstract}
Background: Denosumab and abiraterone were approved by the United States Food and Drug Administration in 2011 for the treatment of metastatic castration-resistant prostate cancer. Neither denosumab nor abiraterone is known to cause rhabdomyolysis.

Case presentation: A 76-year-old Caucasian man with metastatic prostate cancer presented with non-oliguric severe acute kidney injury (AKI) 3 weeks after receiving simultaneous therapy with denosumab and abiraterone. The patient had been on statin therapy for more than 1 year with no recent dose adjustments. His physical exam was unremarkable. Blood work on admission revealed hyperkalemia, mild metabolic acidosis, hypocalcemia, and elevated creatine kinase (CK) at 44,476 IU/L. Kidney biopsy confirmed the diagnosis of rhabdomyolysis-induced AKI. The patient responded well to intravenous isotonic fluids and discontinuation of denosumab, abiraterone, and rosuvastatin, with normalization of CK and recovery of kidney function.

Conclusion: We report the first case of biopsy-proven rhabdomyolysis-induced AKI in a cancer patient acutely exposed to denosumab and abiraterone. Whether one of these drugs individually, or the combination, was the bona fide culprit of muscle breakdown is unknown. Nonetheless, our report is hypothesis-generating for further investigations on the effect of these drugs on muscle cells.
\end{abstract}

Keywords: Denosumab, Abiraterone, Acute kidney injury, Rhabdomyolysis

\section{Background}

Rhabdomyolysis is characterized by muscle breakdown leading to leakage of sarcoplasmic proteins, electrolytes, and myoglobin into the circulation [1]. Several provoking factors (Table 1) can trigger muscle cell death through direct sarcolemmic injury or depletion of adenosine triphosphate within the myocyte [2]. The final common pathway is an increase in intracellular calcium and consequent protease activation, mitochondrial dysfunction, and production of reactive oxygen species that precipitate muscle cell death [3, 4].

One of the common complications of rhabdomyolysis is acute kidney injury (AKI). In the US, rhabdomyolysis

\footnotetext{
* Correspondence: Javier.NeyraLozano@UTSouthwestern.edu

1 Department of Medicine, University of Texas Southwestern Medical Center, 5323 Harry Hines Blvd, Dallas, TX 75390-8516, USA

2Division of Nephrology, University of Texas Southwestern Medical Center, 5323 Harry Hines Blvd, Dallas, TX 75390-8516, USA

Full list of author information is available at the end of the article
}

is the cause of up to $10 \%$ of all cases of AKI [5] and the mortality rate associated with rhabdomyolysis-induced AKI can be as high as $30 \%$, depending on subjects' comorbidities [6]. The occurrence of AKI in rhabdomyolysis is likely from a combination of risk factors that include volume depletion, intrarenal vasoconstriction, direct and ischemic proximal tubular injury (myoglobin-driven), and tubular obstruction [7]. The latter mainly occurs in the distal tubules where myoglobin interacts with TammHorsfall protein, particularly in acidic urine [1, 7].

Pharmacologic agents constitute important causes of non-exertional and non-traumatic rhabdomyolysis [6, 8]. Several drugs such as antipsychotics, statins, and selective serotonin reuptake inhibitors have been identified as common culprits of rhabdomyolysis [6,9], particularly in combination with other patient-specific risk factors.

In 2011, denosumab and abiraterone were approved by the United States Food and Drug Administration for the treatment of metastatic castration-resistant prostate 
Table 1 Identifiable risk factors for rhabdomyolysis

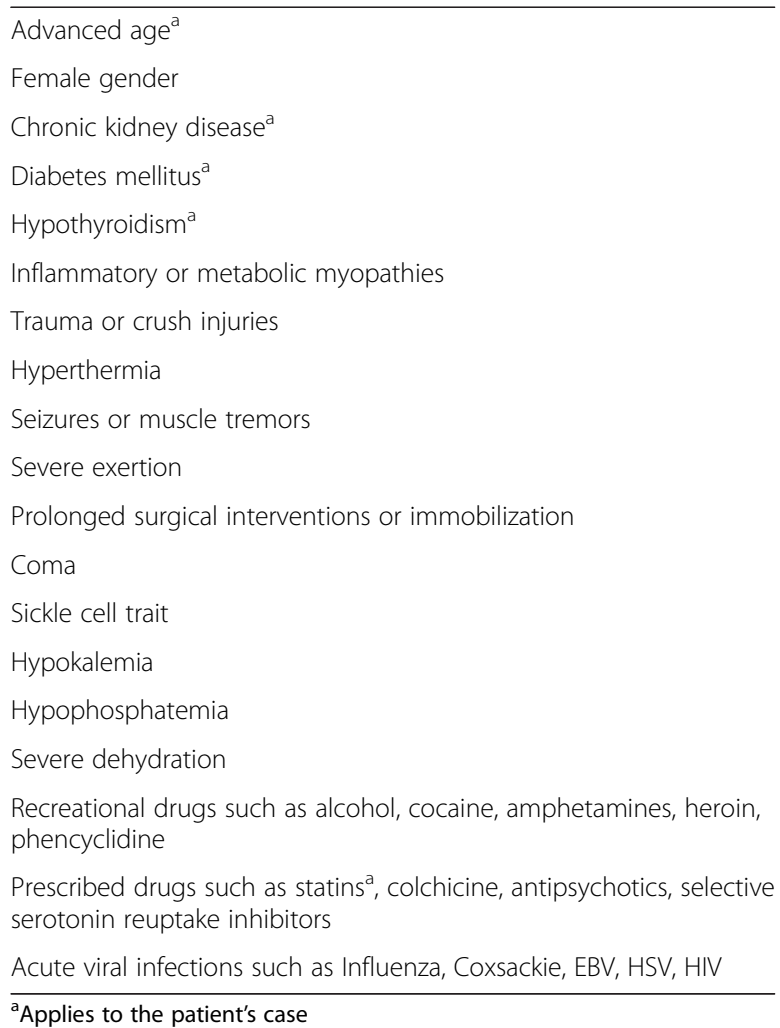

cancer. We present the case of a 76-year-old Caucasian man with a history of metastatic prostate cancer who developed rhabdomyolysis-induced AKI after acute exposure to denosumab and abiraterone.

\section{Case presentation}

A 76-year-old Caucasian man with a history of type 2 diabetes, chronic kidney disease (CKD) stage 3A, essential hypertension, hypothyroidism, antiphospholipid antibody syndrome, prior cerebellar strokes, and prostate cancer (Gleason 10) with widespread metastasis to the bone presented with non-oliguric severe AKI 3 weeks after receiving simultaneous therapy with denosumab (120 mg subcutaneous injection once) and abiraterone ( $1 \mathrm{~g}$ per day orally). The patient had failed prior antineoplastic therapy with leuprolide acetate, bicalutamide, and nilutamide. On admission, his serum creatinine ( $\mathrm{SCr}$ ) was elevated at $5.7 \mathrm{mg} / \mathrm{dL}$ from a baseline of $1.2 \mathrm{mg} / \mathrm{dL}$ (Fig. 1). His active outpatient medications consisted of rosuvastatin (40 mg daily), benazepril, metoprolol tartrate, metformin, warfarin, low-dose prednisone (started concomitantly with abiraterone), and levothyroxine. The patient had been on statin therapy for more than 1 year and the dose had not been recently modified. The patient denied prior episodes of myopathies, rhabdomyolysis, or AKI. His physical exam was unremarkable. Further blood work showed hyperkalemia, mild metabolic acidosis, hypocalcemia, mild transaminemia (predominantly AST), and creatine kinase (CK) of 44,476 IU/L (Table 2). Urine studies revealed dipstick proteinuria $(100 \mathrm{mg} / \mathrm{dL})$, large dipstick blood, only a few normomorphic erythrocytes, and negative culture. All serologic work-up (ANA, PR3- and MPO-ANCA, anti-GBM antibodies, ENA panel, C3, C4, RF) and viral studies (HBV, HCV, CMV, EBV, Influenza A/B, Parainfluenza, Adenovirus, RSV) were negative or normal. Thyroid function tests were normal. Kidney sonogram and Doppler studies were negative for hydronephrosis and renal vein thrombosis, respectively. On admission, abiraterone therapy was discontinued and no further doses of denosumab were administered. Subsequently, the patient underwent kidney biopsy that was consistent with severe acute tubular injury with presence of myoglobin casts, confirming the diagnosis of rhabdomyolysis-induced AKI (Figs. 2 and 3). The patient responded well to intravenous isotonic fluids and discontinuation of denosumab, abiraterone, and rosuvastatin. CK levels normalized by day 25 of hospitalization and the $\mathrm{SCr}$ at the time of hospital discharge was $3.1 \mathrm{mg} / \mathrm{dL}$ (Fig. 1). After hospital discharge, the patient resumed all prior medications, including rosuvastatin, except for denosumab and abiraterone. His successive antineoplastic therapy consisted of enzalutamide. Kidney function returned to baseline 12 months after discharge and he had no recurrent episodes of rhabdomyolysis.

\section{Conclusion}

We present a case of a patient with biopsy-proven AKI caused by rhabdomyolysis 3 weeks after starting simultaneous treatment with denosumab and abiraterone. The patient was also taking rosuvastatin, a drug known to cause rhabdomyolysis [10]. However, his statin exposure was chronic and without reported recent dose adjustments. Most importantly, no other identifiable patientspecific triggers for statin-related rhabdomyolysis (e.g., decompensated hypothyroidism, liver disease, strenuous exercise, or recreational drug use) were identified upon interrogation and examination. In addition, his statin therapy was resumed after discharge with good tolerability thereafter. Nonetheless, it is not known if drug-drug interactions between the statin and denosumab or abiraterone could augment the risk for rhabdomyolysis in humans.

Denosumab is a human monoclonal antibody that neutralizes the receptor activator of nuclear factor kappa-B ligand (RANKL), inhibiting osteoclast-mediated bone resorption [11]. It is used to prevent bone metastasis and skeletal-related events in metastatic castrationresistant prostate cancer $[12,13]$. Some of the common side effects of denosumab include back pain, cystitis, and upper respiratory tract infections. Serious adverse 


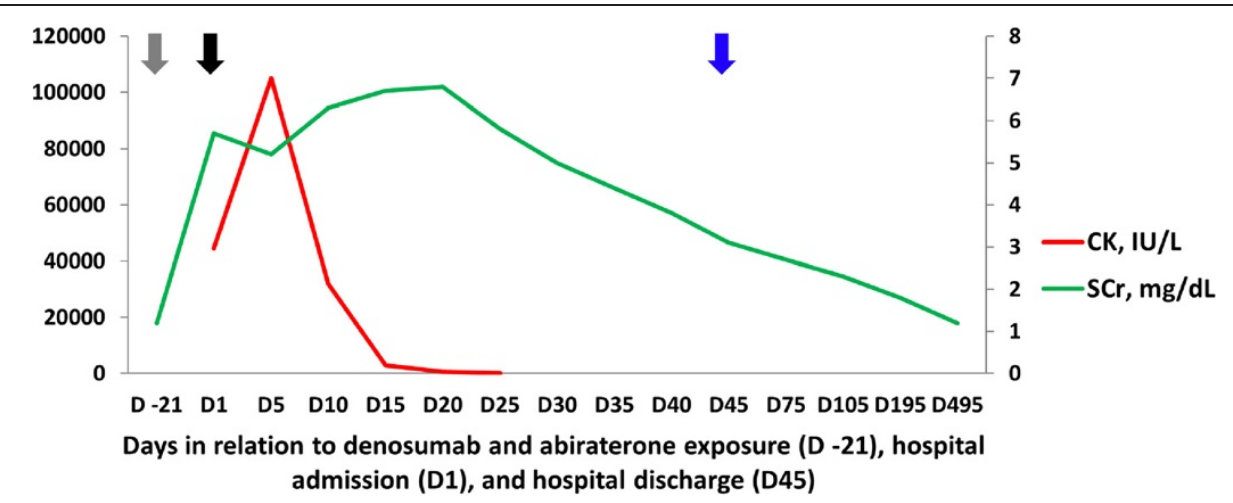

Fig. 1 Time-related serum creatinine ( $\mathrm{SCr}$ ) and creatine kinase (CK) changes in relation to denosumab and abiraterone exposure (D -21), hospital admission (D1), and hospital discharge (D45). Gray arrow = denosumab $120 \mathrm{mg}$ subcutaneous injection (single shot) and abiraterone started at $1 \mathrm{~g}$ orally per day. Black arrow = admission to the hospital, abiraterone stopped. Blue arrow = hospital discharge

events such as osteonecrosis of the jaw have been reported in only $2 \%$ of patients $[14,15]$. Abiraterone is a CYP17A1 inhibitor that blocks the synthesis of androgens in prostate tumor cells, the testes, and adrenal glands. Its side effects are related to 17 -alpha hydroxylase inhibition leading to a decrease in cortisol production and consequent increase in $\mathrm{ACTH}$ release, mineralocorticoid production, and ensuing hypokalemia, which itself is a risk factor for rhabdomyolysis $[16,17]$.

Prior to the development of abiraterone and its approval as an adrenal androgen synthesis inhibitor, ketoconazole was the only CYP17 inhibitor that was used to treat metastatic castration-resistant prostate cancer [18]. However, due to its actions on CYP3A and CYP24A1 and therefore

Table 2 Urinary and blood biochemical profiles at baseline, time of hospital admission, and at the time of peak creatine kinase (CK)

\begin{tabular}{|c|c|c|c|}
\hline & Baseline (before admission) & At the time of admission & At the time of peak $\mathrm{CK}$ \\
\hline \multicolumn{4}{|l|}{ Blood studies } \\
\hline Sodium, mmol/L & $135[135-145]$ & 137 & $134(L)$ \\
\hline Potassium, mmol/L & $4.6[3.6-5.0]$ & $6.2(H)$ & 3.7 \\
\hline Phosphorus, mg/dL & $2.9[2.4-4.5]$ & 2.4 & 2.5 \\
\hline Calcium, mg/dL & $10[8.4-10.2]$ & $8.6(L)$ & $5.7(\mathrm{~L})$ \\
\hline lonized calcium, mg/dL & $-[4.5-5.3]$ & - & $3.4(\mathrm{~L})$ \\
\hline Bicarbonate, $\mathrm{mmol} / \mathrm{L}$ & $29[22-31]$ & $21(L)$ & $13(\mathrm{~L})$ \\
\hline Aspartate aminotransferase, $\mathrm{U} / \mathrm{L}$ & $29[10-50]$ & $354(H)$ & $1423(H)$ \\
\hline Alanine aminotransferase, $\mathrm{U} / \mathrm{L}$ & 19 [10-50] & $125(H)$ & $330(H)$ \\
\hline Creatine kinase, IU/L & $-[39-308]$ & $44,476(H)$ & $105,120(H)$ \\
\hline Lactate dehydrogenase, U/L & $-[135-225]$ & $943(H)$ & $1135(H)$ \\
\hline Creatinine, $\mathrm{mg} / \mathrm{dL}$ & $1.2[0.67-1.17]$ & $5.7(H)$ & $5.2(H)$ \\
\hline eGFR, mL/min/1.73 m $\mathrm{m}^{2 \mathrm{a}}$ & 59 & $10(\mathrm{~L})$ & $11(\mathrm{~L})$ \\
\hline \multicolumn{4}{|l|}{ Urine studies } \\
\hline $\mathrm{pH}$ & $-[5.0-6.0]$ & 6 & 6 \\
\hline Dipstick blood & - [negative] & Large & Large \\
\hline Dipstick protein, mg/dL & - [negative] & 100 & 100 \\
\hline Specific gravity & $-[1.008-1.030]$ & 1.018 & 1.013 \\
\hline Red blood cells/HPF & $-[0-3 / \mathrm{HPF}]$ & 15 & 35 \\
\hline White blood cells/HPF & $-[0-5 / \mathrm{HPF}]$ & $<1$ & 5 \\
\hline Squamous epithelial cells/HPF & $-[0-5 / \mathrm{HPF}]$ & 10 & $<1$ \\
\hline Leukocyte esterase & - [negative] & Negative & Negative \\
\hline
\end{tabular}




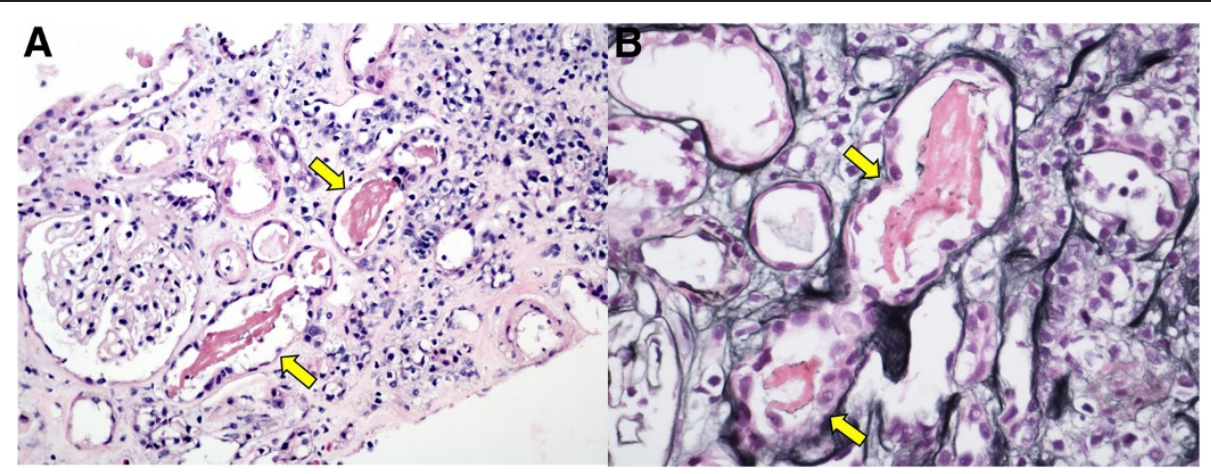

Fig. 2 Kidney biopsy light microscopy. a Hematoxylin \& eosin (X200) showing dark pink, filamentous tubular casts (yellow arrow); b) Jones' silver stain (X400) showing dark pink, coarsely granular and filamentous tubular casts (yellow arrow)

the potential for drug-drug interactions, its use was limited to low-risk clinical scenarios [19]. A few cases of rhabdomyolysis with the concomitant use of ketoconazole and statin have been reported in the literature [20]. In this context, the risk of rhabdomyolysis is thought to be related to the inhibition of CYP3A by ketoconazole, precipitating a significant increase in the plasma concentration of statin. In contrast, abiraterone is known to act only on CYP17, with no apparent interference with statin metabolism. According to the product information, abiraterone metabolites inhibit the hepatic uptake transporter OATP1B1 in vitro, which may theoretically affect OATP1B1-mediated uptake of rosuvastatin, increase its levels, and therefore predispose to toxicity [21-23]. To our knowledge, there are no reported cases of rhabdomyolysis with either acute or chronic exposure to abiraterone, alone or in conjunction with rosuvastatin.

Over the past few years, genetic experiments have unveiled numerous functions of the RANKL/RANK system

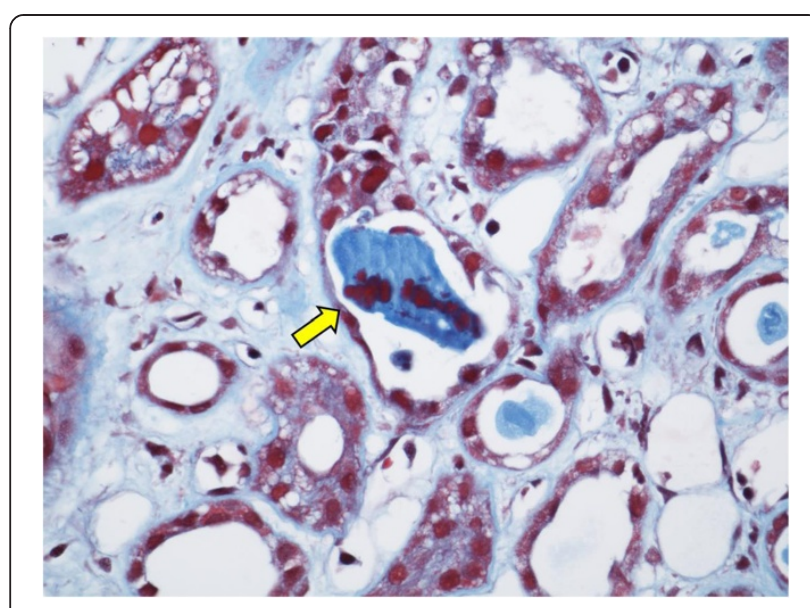

Fig. 3 Kidney biopsy light microscopy showing tubular casts staining positively for myoglobin by immunohistochemistry (anti-myoglobin) (yellow arrow), (X400)
[11]. The best-known role of RANKL/RANK signaling is in bone. Genetic ablation of RANKL or RANK in mice results in a complete lack of osteoclasts, with consequent osteopetrosis and defective tooth eruption [11]. Indeed, inhibition of RANKL/RANK function in osteoclast formation, survival, and function underlies the therapeutic efficacy of denosumab in the treatment of osteoporosis [24]. RANKL also participates in the complex interplay of cancer cells with bone during bone metastasis, by promoting osteoclast formation and thus providing for establishment and growth of bone metastases [25]. These actions of RANKL underlie the efficacy of denosumab in ameliorating skeletal complications in metastatic cancer $[12,13]$. However, animal studies have also revealed roles of this signaling pathway in extraskeletal tissues, such as in lymph node and immune cell development, and in mammary gland development and progestin-driven breast cancer [26]. Hypothalamic RANKL/RANK signaling also plays a key role in thermoregulation and fever [27]. Thus, roles for RANKL/RANK signaling have been found in bone, mammary tissue, the immune system, and CNS, suggesting pleiotropic effects on numerous cell and tissue types.

RANKL mRNA and protein are found in skeletal muscle [28]. However, its function in skeletal muscle is currently unknown, and awaits further studies with tissue-specific knockouts. Therefore, the possible effects of denosumab on skeletal muscle resulting in rhabdomyolysis remain speculative at this time. Possible mechanisms include a direct myopathic effect or alterations in calcium dynamics in the muscle cell, leading to an increase in intracellular calcium concentration and consequent rhabdomyolysis.

We report the first case of rhabdomyolysis leading to severe AKI in association with acute exposure to denosumab and abiraterone. From our case we are unable to determine whether rhabdomyolysis was caused by one of these two drugs, their mutual interaction, or their interaction with another commonly used drug, rosuvastatin. Nonetheless, this case exposes the need to carefully 
monitor patients exposed to denosumab and abiraterone to recognize potential severe adverse effects associated with these novel therapies. In addition, our report is hypothesis-generating to further investigate the effect of these drugs on muscle cells. In conclusion, we recommend monitoring for early signs of rhabdomyolysis in high-risk patients exposed to either denosumab or abiraterone, particularly if other identifiable risk factors are present (Table 1), such as in the case of our patient (e.g., advanced age, CKD, type 2 diabetes, hypothyroidism, and statin use).

\section{Consent}

The patient was deceased at the time of preparation of this manuscript. Written informed consent was obtained from his next-of-kin for publication of this Case Report and any accompanying images.

\begin{abstract}
Abbreviations
ACTH: Adrenocorticotropic hormone; AKI: Acute kidney injury; ANA: Antinuclear antibody; AST: Aspartate aminotransferase; CK: Creatine kinase; CKD: Chronic kidney disease; CMV: Cytomegalovirus; CNS: Central nervous system; CYP: Cytochrome; EBV: Epstein-Barr virus; HBV: Hepatitis B virus; HCV: Hepatitis C virus; mRNA: Messenger RNA; RANKL: Receptor activator of nuclear factor kappa-B ligand; RSV: Respiratory syncytial virus; SCr: Serum creatinine.
\end{abstract}

\section{Competing interests}

A.R.R. has received a speaker's fee from Eli Lilly that was not related to this case report. The remaining authors declare that they have no competing interests.

\section{Authors' contributions}

Analysis of patient's clinical course and outcomes: JAN, NDAR, OUV, and ARR; drafting of the manuscript: JAN, NDAR, and RB; critical revision of the manuscript for important intellectual content: JAN and ARR; critical revision of kidney biopsy results and images: $\mathrm{ARH}$. Administrative, technical, and material support: JAN, NDAR, ARH, and ARR. All authors read and approved the final manuscript.

\section{Acknowledgements}

J.A.N. is supported by the Ben J. Lipps Research Fellowship Program of American Society of Nephrology Foundation for Kidney Research and the Truelson Fellowship Fund at UT Southwestern Charles and Jane Pak Center of Mineral Metabolism and Clinical Research. A.R.R. is supported by the NIDDK (DK091316) and the American Society of Nephrology Gottschalk Award. The authors would like to thank TJ Martin for thoughtful comments on the manuscript.

\section{Author details}

${ }^{1}$ Department of Medicine, University of Texas Southwestern Medical Center, 5323 Harry Hines Blvd, Dallas, TX 75390-8516, USA. ²Division of Nephrology, University of Texas Southwestern Medical Center, 5323 Harry Hines Blvd, Dallas, TX 75390-8516, USA. ${ }^{3}$ Department of Pathology, University of Texas Southwestern Medical Center, 5323 Harry Hines Blvd, Dallas, TX 75390-8516, USA. ${ }^{4}$ Department of Medicine, University of Missouri-Kansas City, Kansas City, Missouri, USA. ${ }^{5}$ Division of Nephrology, University of Missouri-Kansas City, Kansas City, Missouri, USA.

Received: 12 April 2015 Accepted: 17 July 2015

Published online: 30 July 2015

\section{References}

1. Bosch X, Poch E, Grau JM. Rhabdomyolysis and acute kidney injury. N Engl J Med. 2009;361(1):62-72.
2. Warren JD, Blumbergs PC, Thompson PD. Rhabdomyolysis: a review. Muscle Nerve. 2002;25(3):332-47.

3. Giannoglou GD, Chatzizisis YS, Misirli G. The syndrome of rhabdomyolysis: pathophysiology and diagnosis. Eur J Intern Med. 2007;18(2):90-100.

4. Wrogemann K, Pena SD. Mitochondrial calcium overload: a general mechanism for cell-necrosis in muscle diseases. Lancet. 1976;1(7961):672-4.

5. Holt SG, Moore KP. Pathogenesis and treatment of renal dysfunction in rhabdomyolysis. Intensive Care Med. 2001;27(5):803-11.

6. Melli G, Chaudhry V, Cornblath DR. Rhabdomyolysis: an evaluation of 475 hospitalized patients. Medicine. 2005;84(6):377-85.

7. Zager RA, Gamelin LM. Pathogenetic mechanisms in experimental hemoglobinuric acute renal failure. Am J Physiol. 1989;256(3 Pt 2):F446-455.

8. Khan FY. Rhabdomyolysis: a review of the literature. Neth J Med. 2009;67(9):272-83.

9. Huerta-Alardin AL, Varon J, Marik PE. Bench-to-bedside review: Rhabdomyolysis - an overview for clinicians. Crit Care (London, England). 2005;9(2):158-69.

10. Martinez-Lopez D, Enriquez R, Sirvent AE, Redondo-Pachon MD, Millan I, Amoros F. Rhabdomyolysis and acute renal failure following hard physical activity in a patient treated with rosuvastatin. Nefrologia. 2012;32(1):127-8.

11. Hanada R, Hanada T, Penninger JM. Physiology and pathophysiology of the RANKL/RANK system. Biol Chem. 2010;391(12):1365-70.

12. Smith MR, Coleman RE, Klotz L, Pittman K, Milecki P, Ng S, et al. Denosumab for the prevention of skeletal complications in metastatic castration-resistant prostate cancer: comparison of skeletal-related events and symptomatic skeletal events. Ann Oncol. 2015;26(2):368-74.

13. Smith MR, Saad F, Oudard S, Shore N, Fizazi K, Sieber P, et al. Denosumab and bone metastasis-free survival in men with nonmetastatic castrationresistant prostate cancer: exploratory analyses by baseline prostate-specific antigen doubling time. J Clin Oncol. 2013;31(30):3800-6.

14. Langdahl BL, Teglbjaerg CS, Ho PR, Chapurlat R, Czerwinski E, Kendler DL, et al. A 24-month Study Evaluating the Efficacy and Safety of Denosumab for the Treatment of Men With Low Bone Mineral Density: Results From the ADAMO Trial. J Clin Endocrinol Metab. 2015;100(4):1335-42.

15. Prommer E. Palliative Oncology: Denosumab. Am J Hosp Palliat Care. 2015;32(5):568-72.

16. Hoy SM. Abiraterone acetate: a review of its use in patients with metastatic castration-resistant prostate cancer. Drugs. 2013;73(18):2077-91.

17. Auchus RJ, Yu MK, Nguyen S, Mundle SD. Use of prednisone with abiraterone acetate in metastatic castration-resistant prostate cancer. Oncologist. 2014;19(12):1231-40.

18. Potter GA, Barrie SE, Jarman M, Rowlands MG. Novel steroidal inhibitors of human cytochrome P45017 alpha (17 alpha-hydroxylase-C17,20-lyase): potential agents for the treatment of prostatic cancer. J Med Chem. 1995:38(13):2463-71.

19. Barrie SE, Potter GA, Goddard PM, Haynes BP, Dowsett M, Jarman M. Pharmacology of novel steroidal inhibitors of cytochrome P450(17) alpha (17 alpha-hydroxylase/C17-20 lyase). J Steroid Biochem Mol Biol. 1994;50(5-6):267-73.

20. Gilad R, Lampl Y. Rhabdomyolysis induced by simvastatin and ketoconazole treatment. Clin Neuropharmacol. 1999;22(5):295-7.

21. van de Steeg $E$, Greupink R, Schreurs M, Nooijen IH, Verhoeckx KC, Hanemaaijer $\mathrm{R}$, et al. Drug-drug interactions between rosuvastatin and oral antidiabetic drugs occurring at the level of OATP1B1. Drug Metab Dispos. 2013;41(3):592-601.

22. Choi JH, Lee MG, Cho JY, Lee JE, Kim KH, Park K. Influence of OATP1B1 genotype on the pharmacokinetics of rosuvastatin in Koreans. Clin Pharmacol Ther. 2008;83(2):251-7.

23. Pasanen MK, Fredrikson H, Neuvonen PJ, Niemi M. Different effects of SLCO1B1 polymorphism on the pharmacokinetics of atorvastatin and rosuvastatin. Clin Pharmacol Ther. 2007;82(6):726-33.

24. Cummings SR, San Martin J, McClung MR, Siris ES, Eastell R, Reid IR, et al. Denosumab for prevention of fractures in postmenopausal women with osteoporosis. N Engl J Med. 2009:361(8):756-65.

25. Sterling JA, Edwards JR, Martin TJ, Mundy GR. Advances in the biology of bone metastasis: how the skeleton affects tumor behavior. Bone. 2011;48(1):6-15.

26. Hanada R, Hanada T, Sigl V, Schramek D, Penninger JM. RANKL/RANKbeyond bones. J Mol Med (Berlin, Germany). 2011;89(7):647-56. 
27. Hanada R, Leibbrandt A, Hanada T, Kitaoka S, Furuyashiki T, Fujihara H, et al. Central control of fever and female body temperature by RANKL/RANK. Nature. 2009;462(7272):505-9.

28. Kartsogiannis V, Zhou H, Horwood NJ, Thomas RJ, Hards DK, Quinn JM, et al. Localization of RANKL (receptor activator of NF kappa B ligand) MRNA and protein in skeletal and extraskeletal tissues. Bone. 1999:25(5):525-34.

Submit your next manuscript to BioMed Central and take full advantage of:

- Convenient online submission

- Thorough peer review

- No space constraints or color figure charges

- Immediate publication on acceptance

- Inclusion in PubMed, CAS, Scopus and Google Scholar

- Research which is freely available for redistribution 